Gut, 1971, 12, 873-877

\title{
Mucosal architecture of the small bowel in cases of psoriasis ${ }^{1}$
}

\author{
R. E. BARRY, P. R. SALMON, A. E. READ, AND R. P. WARIN \\ From the Department of Medicine, Bristol Royal Infirmary
}

SUMMARY Using a strictly defined grading system to eliminate subjective assessment of mucosal architecture, the jejunal mucosa from cases of extensive psoriasis has been shown to be significantly different from that of normal controls obtained both in life and at postmortem examination. The same changes, however, are shown to occur in patients who are ill and losing weight for reasons other than psoriasis.

Diminished lactose utilization, demonstrated in extensive psoriasis, supports the suggestion of a decreased mucosal surface area in this disease.

The concept of dermatogenic enteropathy was introduced by Shuster and Marks (1965) following their observation that steatorrhoea occurring in nine out of 10 patients with widespread skin disease, including psoriasis and eczema, improved following treatment of the skin alone. Subsequently they showed a relationship between the area of skin involved and fat malabsorption (Shuster, Watson, and Marks, 1967). Their initial observations demonstrated a structural abnormality of the jejunal mucosa in psoriasis (Shuster et al, 1967).

Recently, however, they have reassessed their data (Marks and Shuster, 1970) and now find no difference between the jejunal mucosa from cases of psoriasis and that from a control population in the Newcastle area. Thus the presence of architectural changes in the jejunal mucosa in psoriasis seems to be in doubt. We have studied 22 cases of extensive psoriasis and compared the findings with control populations in the Bristol area.

\section{Selection of Patients}

Three groups of patients were studied, consisting of one group of patients with psoriasis and two control groups.

GROUP 1

This group consisted of 22 cases of psoriasis, the sole criterion for selection being that they were admitted to the Bristol Royal Infirmary with more than $50 \%$ ${ }^{1}$ Based on a paper read to the British Society of Gastroenterology in April 1971.

Received for publication 2 September 1971. body surface involved. Only one patient gave a history of frequent, loose bowel actions.

\section{GROUP 2}

Group 2 consisted of (a) 20 normal controls matched with group 1 for age but not sex. These were patients admitted to hospital for the investigation of vague abdominal symptoms in whom all tests of small bowel function were normal and in whom no organic disease could be found; (b) 36 cases coming to necropsy after sudden death, ie, after illnesses of sudden onset and less than 24 hours' duration, and included ruptured ectopic pregnancies, road traffic accidents, dissecting aneurysms, and similar catastrophes. These patients were asymptomatic before their sudden deaths.

\section{GROUP 3}

This group consisted of $(a) 15$ patients who were ill and had lost more than 2 stone $(28 \mathrm{lb})$ in weight for reasons other than skin or gastrointestinal disease; (b) 52 patients who had come to necropsy after illnesses lasting more than three days and who had lost more than 2 stones $(28 \mathrm{lb})$ in weight. This group suffered from such diseases as chronic heart failure, anorexia nervosa, collagen disease, and chronic sepsis.

\section{Methods}

Peroral jejunal biopsies were obtained from the patient volunteers using a Watson capsule screened to a point in the jejunum $6 \mathrm{in}$. distal to the ligament of Treitz. The stereomicroscope features of each 


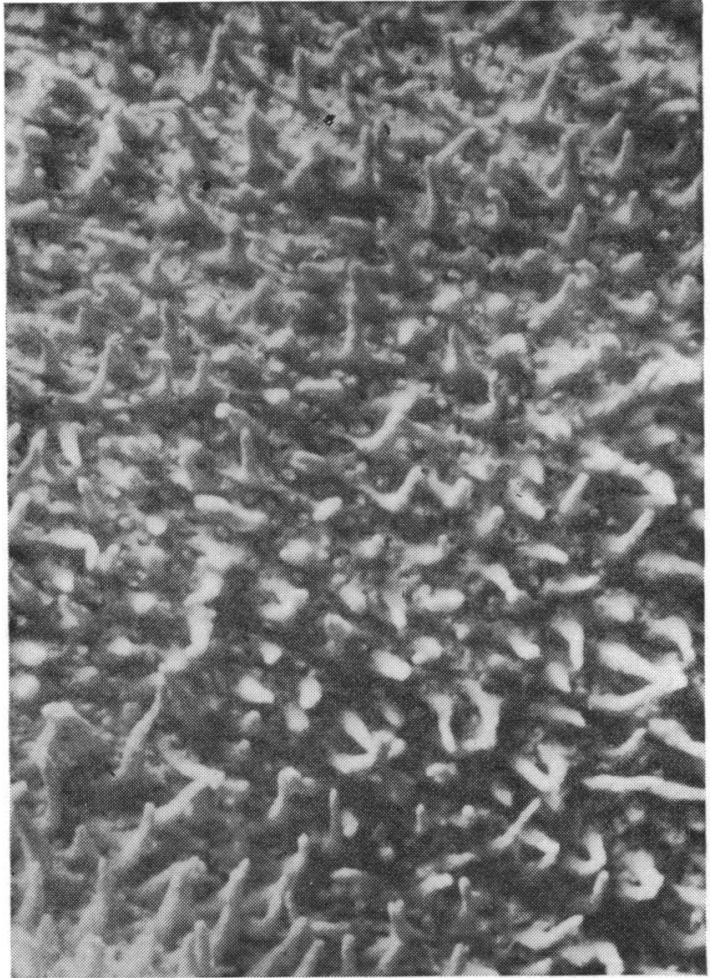

Fig. 1 The presence of cylindrically shaped villi only makes this postmurtem preparation grade 1 . The villi appear thin and sparse because, having washed away the epithelium after autolysis, there remains only the skeleton of the lamina propria.

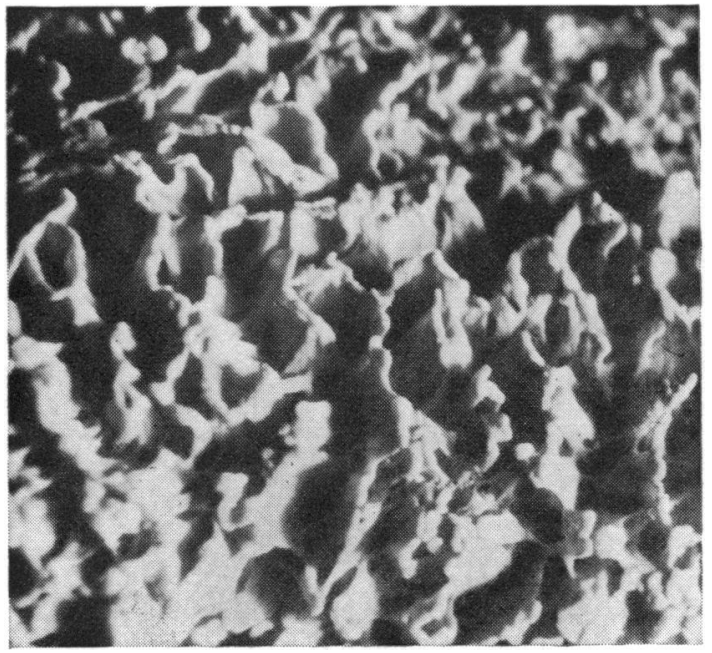

Fig. 3 Postmortem preparation grade 3.

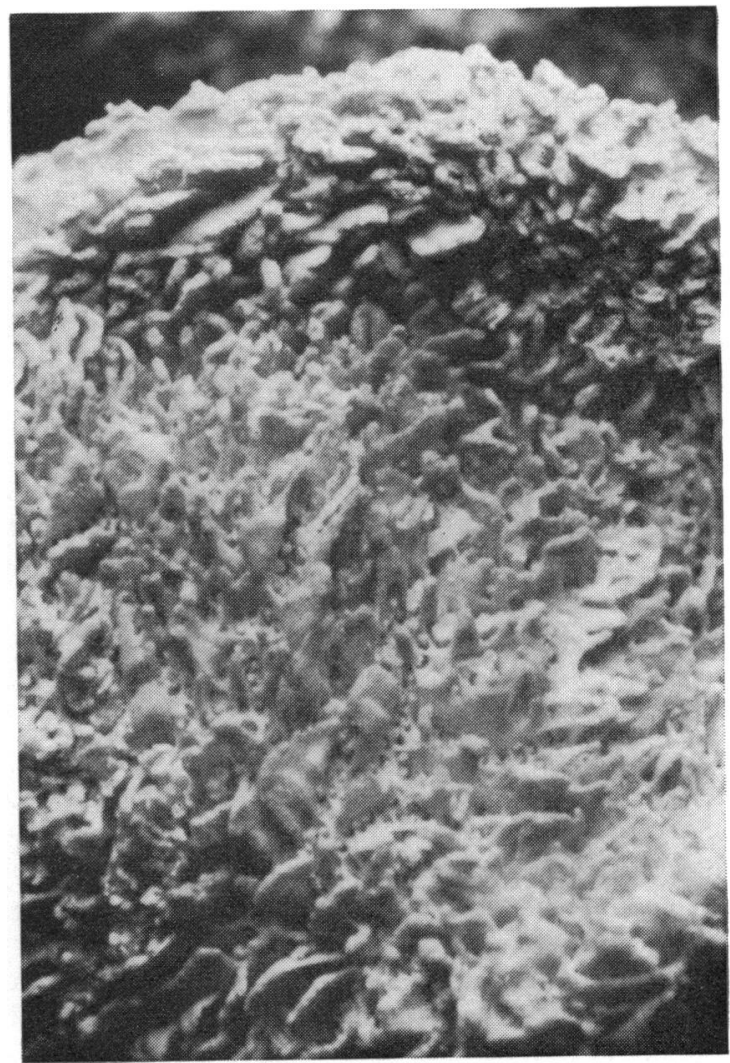

Fig. 2 Postmortem preparation grade 2.

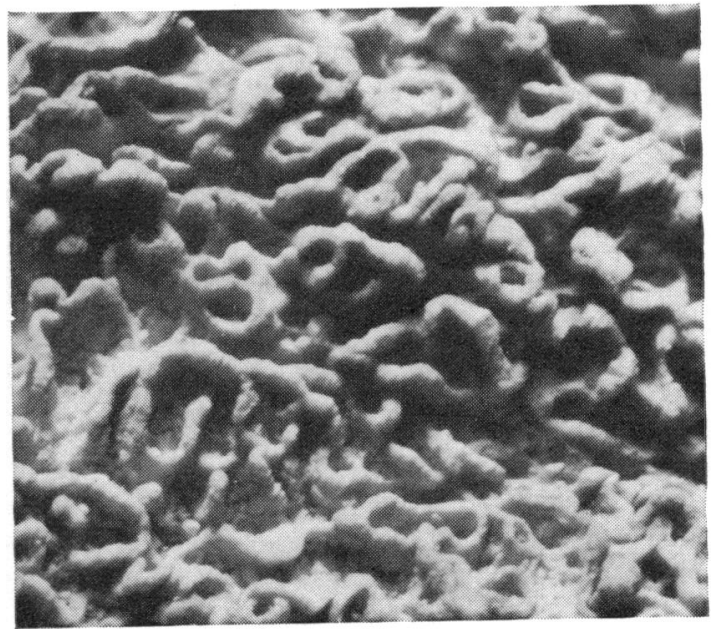

Fig. 4 Postmortem preparation grade 4. 
biopsy were recorded photographically before each specimen was processed for histological section.

The mucosal architecture of jejunal biopsies is usually described according to the dominant mucosal feature, eg, convolutions. Whereas this causes no difficulty in the majority of biopsies, it does involve a subjective assessment of the features which is not always easy to make. To overcome this problem we have used a grading system so defined as to eliminate the need for a subjective assessment. The architectural features of each biopsy are graded according to the presence of the following architectural features.

GRADE 1

Cylindrically shaped villi only are present.

GRADE 2

Leaf-shaped villi are present. A leaf is defined as having a long diameter greater than twice the shorter diameter.

\section{GRADE 3}

Ridged-shaped villi are present. A ridge is defined as having a long diameter more than 10 times the shorter.

\section{GRADE 4}

Convolutions are present. A convolution is defined as a ridge containing a minimum of two angulations of $90^{\circ}$ or less.

In the postmortem populations studied, a piece of intestinal mucosa approximately 1 in. square was obtained from a site 6 in. distal to the ligament of Treitz and then autolysed in isotonic saline overnight before fixation. It was then dehydrated and stained according to the method of Loehry and Creamer (1966) so as to render visible the antemortem mucosal architecture, which could then be graded in the same way as illustrated in Figures 1-4. Study of jejunal mucosa before and after autolysis shows that this process does not affect the grading.

Faecal fat excretion and xylose tolerance tests (Sammons, Morgan, Frazer, Montgomery, and Phillips, 1967) were performed on all live cases included in the study, and a lactose utilization test (Salmon, McCarthy, and Read, 1969; Ueda, 1970) was performed on 15 cases of psoriasis.

\section{Results}

The incidence of the various mucosal features in fresh peroral biopsies is shown in Figure 5. The results in the normal population agree closely with those found by Marks and Shuster (1970), in particular the unexpected incidence of grade 4 (convolutions).

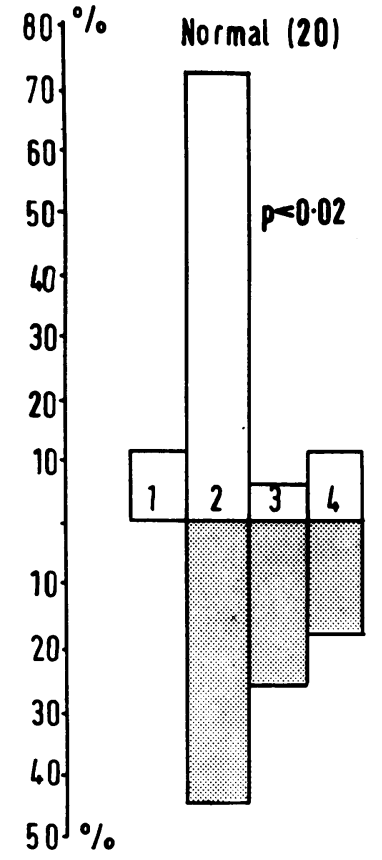

Psoriasis (22)
Illness (15)

N.S.

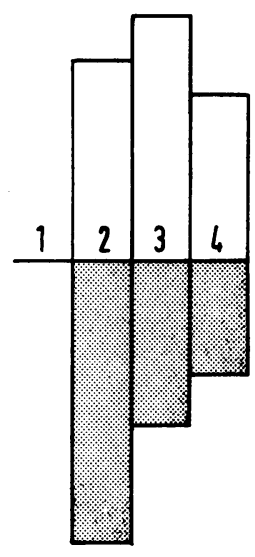

Psoriasis (22)
Fig. 5 The incidence of mucosal features in fresh peroral biopsies.

In psoriasis, however, the distribution curve is displaced to the right, ie, there is a higher incidence of grades 3 and 4, there being less than half of the cases of psoriasis with biopsies of grade 2 or less. This finding is significant at the level of 1 in 50 .

When, however, the findings in psoriasis are compared with those in wasting diseases (Fig. 5), the distribution curves are very similar with a high incidence of grades 3 and 4 and a low incidence of grades 1 and 2 . There is, in fact, no statistical difference between these two groups.

A similar pattern was found in the postmortem populations studied (Fig. 6). As expected, patients dying suddenly have mucosal patterns similar to the normal group of fresh peroral biopsies. Patients dying of wasting diseases of more than three days' duration show small bowel mucosal changes similar to those found in peroral biopsies in psoriasis.

Of the 15 patients with psoriasis studied by means of the lactose utilization test, the mean two-hour excretion rate of radiocarbon dioxide was $6.29 \% \pm$ $2 \cdot 37$ which was significantly lower than the normal controls $(8.22 \% \pm 1.99$;) $\mathrm{P}<0.01$. A significant correlation was found between patients with a large area of skin involved and failure to utilize lactose${ }^{1-}{ }^{14} \mathrm{C}$ (Fig. 7). Intravenous glucose tolerance tests performed on 10 of these patients and $K$ values 


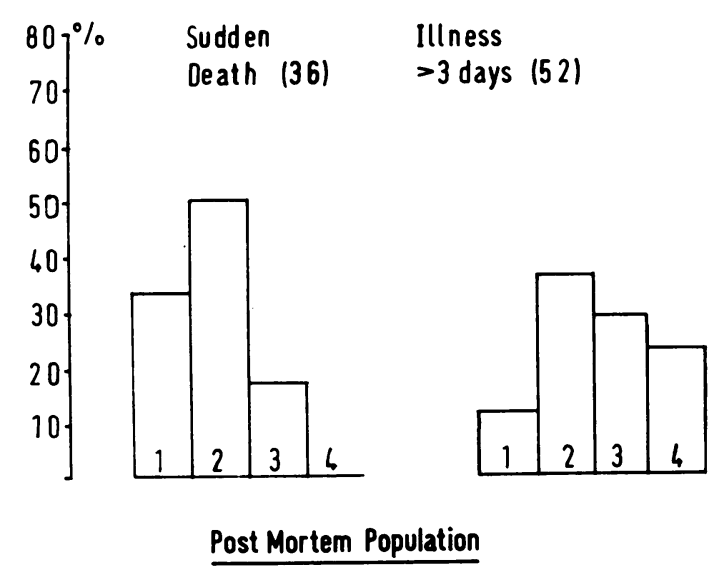

Fig. 6 The incidence of mucosal features in the postmortem populations.

calculated according to the method of Lundbaek (1962) demonstrated that there was no impairment of glucose tolerance. The additional finding of normal D-xylose excretion in 20 out of 22 patients suggests that these results are not due to a defect of monosaccharide absorption, but that patients with extensive psoriasis have diminished intestinal lactase.

In the two patients with abnormal D-xylose excretion there was also steatorrhoea in association with biopsies of grades 3 and 4 respectively. The area of skin involved showed no correlation with the mucosal findings or any parameter in this study other than with lactose utilization.

\section{Discussion}

The results show that there is a significant difference in the mucosal architectural features in the jejunum of patients with psoriasis when compared with normal controls. It is also clear, however, that precisely the same changes occur in patients with wasting disease.

The postmortem and peroral specimens are not strictly comparable because the difference in their size may affect the grading and it is for this reason that the data from these sources are presented separately and not pooled. There are, of course, no postmortem data on patients with psoriasis who do not normally come to postmortem examination.

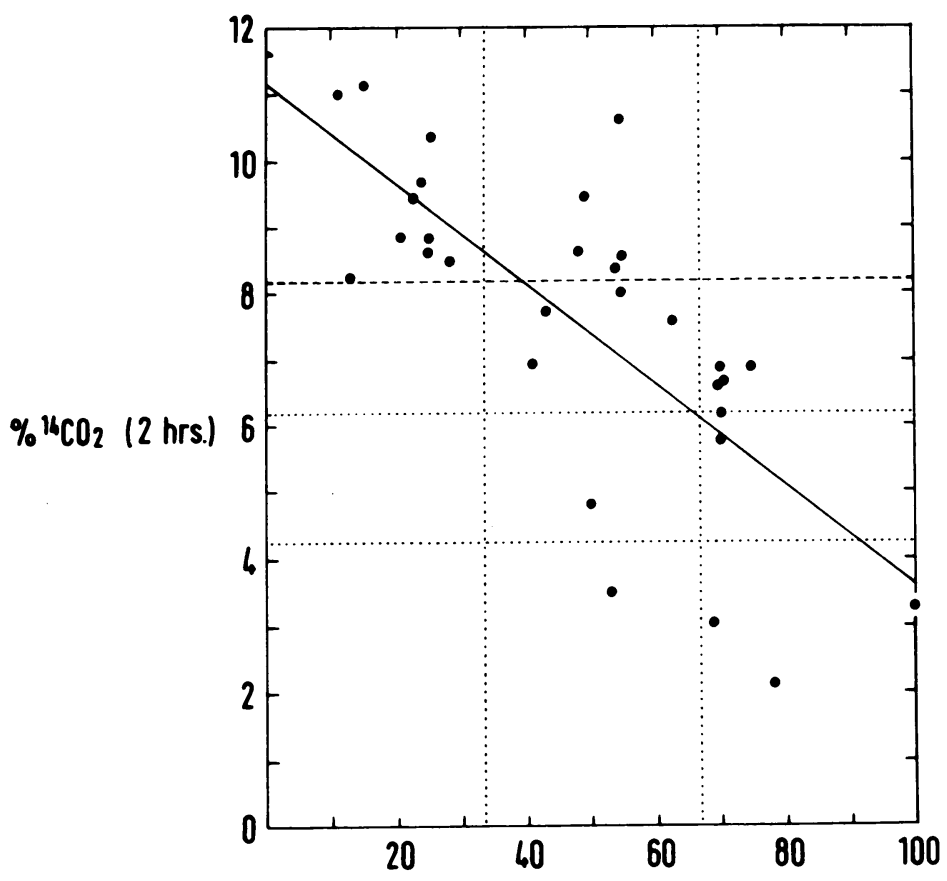

Fig. 7 The correlation between lactose utilization with the extent of the rash.

Extent of rash: \% body surface involved

$$
y=-0.0749 x+11.1769 \quad r=-0.725 \quad P<0.001
$$


Thus the postmortem data indicate that the mucosa in non-specific illness differs from normal.

Both the structural and functional intestinal changes described suggest that there is a decrease in the small bowel surface area in patients with severe psoriasis. The fact that lactose utilization correlates with the extent of the rash when the presence of convolutions does not is difficult to explain but may reflect the fact that this test is a measure of the total lactose available throughout the whole of the small bowel and, as such, is more likely to represent a true measure of surface area than may be expected from consideration of a small biopsy specimen taken from a single locality.

The results presented here differ from those found in the Newcastle population (Marks and Shuster, 1970). It seems probable that this difference is due to the criteria for selection of the patients studied rather than to a regional variation in mucosal architecture. In the present study, psoriasis was the only skin disease investigated and the extent of the disease (greater than $50 \%$ surface area involved) was greater than in the Newcastle series.

The incidence of mucosal changes in illnesses other than psoriasis means that only those subjects known to be perfectly healthy, not merely free from psoriasis, may be expected to have a normal jejunal mucosa. The apparent high incidence of convolutions in the Newcastle series may be due, in part, to inclusion in their postmortem control group of patients who had died after lengthy illnesses which may, in themselves, result in architectural changes.

The unexpected incidence of grade 4 biopsies in the 'normal' peroral biopsy group presented here may reflect the fact that the subjects studied were symptomatic, ie, although no evidence of organic disease could be found, the very fact that this group were patients in hospital may indicate that they are less likely to be truely 'normal' than is an asymptomatic man in the street. Because no evidence of disease could be found does not of nesessity mean that it does not exist. The selection of 'normal' cases for any clinical study may be exceedingly difficult and must rest in the final analysis on the definition of 'normality' which is acceptable for that study. The equivalent normal group in the postmortem study (sudden death group), who were known to be asymptomatic before their sudden death, did not show this incidence of grade 4 features although the two groups were statistically indistinguishable.

The results indicate that changes in the small bowel mucosal architecture do indeed occur in psoriasis but that they also occur in patients who are ill and losing weight from other causes. Thus structural changes do exist in psoriasis but there would appear to be no reason to regard it as specifically dermatogenic.

We are indebted to Dr R. P. Warin and Dr C. D. Evans of the Department of Dermatology for their help and encouragement in the study of their patients.

\section{References}

Loehry, C. A., and Creamer, B. (1966). Postmortem study of smallintestinal mucosa. Brit. Med, J., 1, 827-829.

Lundbaek, K. (1962). Intravenous glucose tolerance test as a tool in definition and diagnosis of diabetes mellitus. Brit. Med. J., 1, 1507-1513.

Marks, J., and Shuster, S. (1970). Small-intestinal mucosal abnormalities in various skin diseases-fact or fancy? Gut, 11, 281-291.

Salmon, P. R., McCarthy, C. F., and Read, A. E. (1969). An isotope technique for measuring lactose absorption. Gut, 10, 685-689.

Salmon, P. R., Read, A. E., and Warin, R. (1969). Radiocarbon estimation of lactose absorption; a survey of 104 patients with skin disease. Gut, 10, 1052.

Sammons, H. G., Morgan, D. B., Frazer, A. C., Montgomery, R. D., Philip, W. M., and Phillips, M. J. (1967). Modification in the xylose absorption test as an index of intestinal function. Gut. 8, 348-353.

Shuster, S., and Marks, J. (1965). Dermatogenic enteropathy; a new cause of steatorrhoea. Lancet, 1, 1367-1368.

Shuster, S., Watson, A. J., and Marks, J. (1967). Small intestine in psoriasis. Brit. Med. J., 3, 458-460.

Ueda, H. (1970). ${ }^{14} \mathrm{C}$ lactose absorption test and lactase deficiency in milk intolerance. In Advance Abstracts, 4th World Congress of Gastroenterology, Copenhagen 1970. 